\title{
NORMA SUBJETIVA, INTENCIÓN Y CONSUMO DE MARIHUANA EN JÓVENES UNIVERSITARIOS DE MÉXICO
}

\section{SUBJECTIVE NORM, INTENTION AND CONSUMPTION OF MARIJUANA IN COLLEGE STUDENTS OF MÉXICO}

\author{
Francisco Rafael Guzmán Facundo* \\ Maura Liliana Llamas Estrada ${ }^{* *}$ \\ Lucio Rodríguez Aguilar ${ }^{* * *}$ \\ María Magdalena Alonso Castillo ${ }^{* * * *}$
}

\begin{abstract}
RESUMEN
Este artículo tuvo como propósito conocer la relación de la norma subjetiva con la intención y el consumo de marihuana en un grupo de jóvenes universitarios del Norte de México. El diseño del estudio fue descriptivocorrelacional, los datos fueron obtenidos a través de la aplicación de un instrumento sobre la Norma Subjetiva para el consumo de marihuana y analizados en el programa SPSS 18. Participaron 558 jóvenes de nueve facultades de una universidad pública. Los resultados mostraron que $15.1 \%$ ha consumido marihuana alguna vez en la vida. La prevalencia del consumo de marihuana fue mayor en hombres $\left(\chi^{2}=42.5, \mathrm{p}<.001\right)$, en mayores de 20 años de edad $\left(\chi^{2}=11.64, \mathrm{p}<.001\right)$ y en los estudiantes de ingenierías y ciencias agropecuarias $\left(\chi^{2}=9.54, \mathrm{p}<.023\right)$. La norma subjetiva se relacionó positivamente con la intención de consumo $\left(r_{s}=.224 ; \mathrm{p}<.001\right)$. La norma subjetiva fue más alta en jóvenes que muestran intención de consumo que aquellos que no tienen intención $(\bar{X}=$ 15.85 y $\bar{X}=6.99 ; U=20561.5, p<.001$ ), de la misma forma la norma subjetiva fue más alta en los jóvenes que han consumido marihuana que en aquellos que nunca han consumido ( $\bar{X}=220.94$ y $\bar{X}=7.05 ; U=28713.5, p<.001)$. Los resultados presentados aportan al conocimiento de la disciplina de enfermería y áreas a fines, principalmente en la línea de generación de conocimientos de prevención de adicciones y en un futuro podrán servir como base para la justificación de diseños de programas preventivos.
\end{abstract}

Palabras clave: Factores de riesgo, intención, Cannabis, abuso de marihuana, estudiantes.

\begin{abstract}
This article was aimed to determine the relationship between subjective norm with intention and marijuana consumption in a group of college students in northern Mexico. The study design was descriptive, co relational; data collection was obtained through the application of a Subjective Norm Instrument for marijuana consumption and analyzed with SPSS 18. Study participants were 558 students from nine faculties of a public university. Results showed that $15.1 \%$ had used marijuana at some time in their life. The prevalence of marijuana consumption was higher in men $\left(\chi^{2}=42.5, \mathrm{p}<.001\right)$, older than 20 years of age $\left(\chi^{2}=11.64, \mathrm{p}<.001\right)$ and students in engineering and agricultural sciences $\left(\chi^{2}=9.54, \mathrm{p}<.023\right)$. The subjective norm is positively related

\footnotetext{
*Académico Cuerpo de Prevención de Adicciones a las Drogas de la Facultad de Enfermería de la Universidad Autónoma de Nuevo León (UANL). México. Email: francisco.guzmanf@uanl.mx.

${ }^{* *}$ Maestra de la Facultad de Enfermería de Mazatlán de la Universidad Autónoma de Sinaloa, México. Email: mauraliliana@ hotmail.com

${ }_{* * *}^{*}$ Académico Cuerpo de Prevención de Adicciones a las Drogas de la Facultad de Enfermería de la Universidad Autónoma de Nuevo León (UANL). México. Email: lucio.rodrigueza@uanl.mx

${ }_{* * * *}^{*}$ Académica Cuerpo de Prevención de Adicciones a las Drogas de la Facultad de Enfermería de la Universidad Autónoma de Nuevo León (UANL). México. Email: magdalena_alonso@hotmail.com
} 
with intention $\left(\mathrm{r}_{\mathrm{s}}=.224, \mathrm{p}<.001\right)$. The subjective norm was higher in young people who displayed intention for consumption than those who have no intention $(\bar{X}=15.85$ and $\bar{X}=6.99, \mathrm{U}=20561.5, \mathrm{p}<.001)$; in the same way, subjective norm was higher in young people that have used marijuana than those who have never used it $(\bar{X}=220.94$ and $\bar{X}=7.05, \mathrm{U}=28713.5, \mathrm{p}<.001)$. The results are a contribution to the discipline of nursing knowledge and related areas, especially in the line of knowledge generation about addiction prevention, and in the future may serve as a basis for the rationale design of preventive programs.

Key words: Risk factors, intention, Cannabis, marijuana abuse, students.

Fecha recepción: 05/01/11 Fecha aceptación: 13/04/12

\section{INTRODUCCIÓN}

El consumo de la marihuana tiene diversas consecuencias sobre la salud, tanto a nivel orgánico como psicológico. A nivel orgánico el consumo crónico se ha asociado con la aparición de bronquitis, tos crónica y cambios precancerosos en el tejido pulmonar. A nivel psicológico, estudios muestran que interfiere en la ejecución de funciones complejas, como la memoria, la concentración o el aprendizaje, puede provocar una disminución del rendimiento escolar en los jóvenes, exacerbar la psicosis y aumentar el riesgo de accidentes de tráfico (1).

A pesar de las consecuencias provocadas, la marihuana es la droga ilegal que se consume con mayor frecuencia en la mayoría de los países. En México se ha incrementado el consumo considerablemente en los últimos años; los resultados de la Encuesta Nacional de Adicciones (ENA) indican incremento de $3.5 \%$ en el 2002 a $4.2 \%$ en el 2008 en la población general. Cabe destacar que la marihuana ha ocupado los primeros lugares de preferencia desde la primera encuesta nacional realizada en el año 1988, mostrando mayor consumo en los hombres que en mujeres (4.6 hombres por cada mujer) y en jóvenes de 18 a 34 años de edad (2).

Los resultados de la ENA muestran que actualmente los jóvenes están más expuestos a oportunidades de usar drogas que las generaciones anteriores. El consumo de drogas es un fenómeno complejo y multifactorial, $y$ es necesario tener en cuenta el comportamiento de los jóvenes, así como sus factores de riesgo; actualmente algunos estudios muestran que los factores asociados al inicio del consumo de drogas son de carácter individual, como la edad, el sexo, tener un bajo rendimiento académico, consumir drogas legales y tener una conducta antisocial; de carácter interpersonal, como el nivel de comunicación, relación con los padres, problemas familiares y la relación con amigos consumidores de drogas, entre otros (3-5).

El proceso de socialización juega un papel importante para el inicio del consumo de drogas, de modo especial el medio familiar y el medio de los iguales. Es en el seno de la familia y los amigos donde la persona se socializa, aprende y adquiere tanto creencias como actitudes, normas sociales interiorizadas y valores, a partir de ello toma decisiones sobre su conducta. El aprendizaje por observación, las consecuencias de las acciones, entre otras, van poco a poco moldeando el modo de ser del niño y posteriormente del adolescente y joven. Se ha documentado que el tener amigos y familiares consumidores de sustancia muestra una influencia directa en el consumo de drogas (6). Un concepto que se forma de la influencia de la familia y los amigos es la norma subjetiva, este concepto surgió originalmente de la Teoría de Acción Razonada y posteriormente retomada en la Teoría de la Conducta Planeada (TCP) (7).

La norma subjetiva es un razonamiento que pone de manifiesto la presión social percibida por la persona que va a realizar la 
conducta. Muestra el resultado de la evaluación que la persona hace si los otros significativos (familiares o amigos) piensan que él o ella realicen la conducta y de las motivaciones para ajustarse a esas expectativas (7). El conocimiento sobre las creencias específicas de lo que los otros piensan de cada uno de los comportamientos específicos como el consumir drogas va a influir en la intención de llevar a cabo o no una conducta general, siempre en función de la motivación para complacerlos. Es decir si una persona percibe que sus amigos o algún familiar piensan o están de acuerdo que él consume alguna droga, su decisión tiende a inclinarse a esa conducta y a su vez estará motivado para complacerlos.

Existe evidencia empírica en la literatura internacional sobre la relación positiva de las normas subjetivas con la intención de consumo de drogas en adolescentes y jóvenes escolarizados. Así mismo se ha reportado que los usuarios de drogas perciben mayores normas subjetivas (8-12). Recientemente en México se ha publicado un artículo que reporta la predicción de los conceptos de la TCP donde se incluye la norma subjetiva, destacando predicciones significativas de las normas subjetivas sobre el consumo de drogas ilícitas en adolescentes de educación media $(13,14)$.

Sin embargo en México poco se ha abordado sobre el concepto de norma subjetiva para el consumo de marihuana en jóvenes, aunado que Sinaloa, México, es el segundo estado donde existe mayor proporción de jóvenes consumidores de marihuana (2), por lo que este estudio tiene gran relevancia al abordar la norma subjetiva para el consumo de marihuana en jóvenes universitarios, dado que los resultados aportarán conocimiento a la disciplina de enfermería y áreas afines, principalmente en la línea de generación de conocimientos de prevención de adicciones, y en un futuro podrán servir como base para la justificación de diseños de programas preventivos. Por lo anterior los objetivos e hipótesis del estudio fueron los siguientes:

\section{Objetivos:}

Describir la prevalencia del consumo de marihuana alguna vez en la vida, en el último año y en los últimos 30 días por sexo, edad, estado laboral y área de estudio en jóvenes universitarios. 2) Analizar la relación de la norma subjetiva con la intención y el consumo de marihuana.

\section{Hipótesis:}

1. Los jóvenes universitarios que muestran intención de consumir marihuana muestran mayor percepción de norma subjetiva que los jóvenes que no refieren intención.

2. Los jóvenes universitarios que han consumido marihuana muestran mayor percepción de norma subjetiva para el consumo de marihuana que los jóvenes que no han consumido marihuana.

\section{MATERIAL Y MÉTODOS}

El diseño de estudio fue descriptivo y correlacional. Para una población de 8.314 jóvenes universitarios hombres y mujeres de una universidad pública de Mazatlán, Sinaloa, México. La determinación del tamaño de la muestra se estimó a través del programa n Query Advisor 4.0, se consideraron dos grupos (consumidores y no consumidores), con un nivel de confianza del 95\% para una prueba unilateral, con efecto mediano de .05 , considerando ocho no consumidores de marihuana por un consumidor, la determinación del tamaño de la muestra inicial fue de 351 , se consideró un efecto de diseño de 1.5 y una tasa de no respuesta de .05 , dando como resultado una muestra final de 558 universitarios distribuidos en 23 grupos.

El muestreo fue aleatorio-estratificado con asignación proporcional al tamaño del estrato; se consideraron nueve estratos for- 
mados por las facultades en estudio, dentro de cada estrato se utilizó muestreo por conglomerados unietápico, donde los grupos formaron el conglomerado. Para medir las variables del estudio se utilizó dos instrumentos y una cédula de datos personales para conocer los datos sociodemográficos; el primer instrumento midió la norma subjetiva e intención para el consumo de marihuana y el segundo midió el consumo de marihuana.

El primer instrumento está basado en los conceptos de la Teoría de la Conducta Planificada, fue elaborado por un grupo de investigadores mexicanos (14), originalmente el instrumento mide actitudes, normas subjetivas, control percibido e intenciones para el consumo de drogas. Para fines de este estudio sólo se tomaron los que corresponden a norma subjetiva e intención (NORSUB). El instrumento de norma subjetiva está compuesto por dos subescalas, la primera corresponde a las creencias normativas que comprende cuatro preguntas con tres opciones de respuesta, falso, ni cierto ni falso y cierto (Ejemplo: ¿Tus mejores amigos o familiares piensan que alguna vez tú podrías probar alguna marihuana?), en este estudio se obtuvo una consistencia interna aceptable $(\alpha=0.80)$. El segundo apartado, que corresponde a la disposición de ajustarse a las creencias normativas, consta de cuatro reactivos con cinco opciones de respuesta que van desde nunca hasta siempre (Ejemplo: ¿Qué tan dispuesto estás a seguir los consejos o hacer lo que esperan de ti, tus amigos o familiares?), obteniendo un Alpha de Cronbach de 0.92.

La forma de evaluación es a través de la creación de índices donde se toman valores de 0 a 100 para cada subescala, posteriormente para calcular el valor general de norma subjetiva se procede a multiplicar el resultado de los índices dividido entre 100, lo cual permite tener valores de 0 a 100, donde se indica que a mayor puntuación mayor son las normas subjetivas para el consumo de marihuana.

Los indicadores empíricos de la intención originalmente están planteados para medir las intenciones para el consumo de drogas ilícitas en general, sin embargo para este estudio se plantearon específicamente para la intención del consumo de marihuana, la cual se midió a partir de dos preguntas con cinco opciones de respuesta, donde se da un valor de 1 a 5 (no es probable, es poco probable, es más o menos probable, es probable y es muy probable), diferenciada para quienes han consumido marihuana y para quienes no lo han hecho ( $\mathrm{Si}$ has consumido marihuana, ¿qué tan probable es que vuelvas a consumir marihuana? Si nunca has probado marihuana, ¿qué tan probable es que llegues a consumir marihuana?). Un valor cerca del cinco indica mayor intención para el consumo de marihuana.

El segundo instrumento es el historial del consumo de marihuana que cuestiona sobre el uso de marihuana alguna vez en la vida, en el último año, en los últimos 30 días, así como número de días de consumo en los últimos treinta días; este instrumento se tomó de la cédula para medir dependencia de drogas publicadas en la Norma Oficial Mexicana para la Prevención, Tratamiento y Control de las Adicciones (15).

Para la selección de los sujetos de estudio se tomó como marco muestral el listado de número de grupos de cada facultad. A cada grupo se le asignó un número, posteriormente se seleccionó de forma aleatoria los grupos correspondientes de cada facultad, una vez identificados los grupos se procedió a su localización, se realizó en horarios de clases y la aplicación de los instrumentos se coordinó junto con la subdirección académica de cada facultad, cuidando interferir lo menos posible en sus horarios de clase.

El presente estudio fue revisado y autorizado por el Comité de Ética e investigación de las instituciones correspondientes. Se programó un día de aplicación de instrumentos 
por facultad, ya identificados los grupos se les entregó en primer lugar el consentimiento informado para su autorización, una vez firmado se procedió a la aplicación de la encuesta. Se orientó a los alumnos a contestar de acuerdo a las instrucciones de cada cuestionario teniendo en cuenta en dar siempre la respuesta más cercana a su realidad, además de que se hizo hincapié en responder a todas las preguntas sin dejar alguna sin respuesta, posteriormente de terminar de contestar el instrumento se colocó en un sobre sellándolo y se depositó en un contenedor especial, cuidando de que no se violara el anonimato de sus respuestas.

Para análisis de datos se utilizó el SPSS (Statistical, Package for the Social Sciences) versión 18. Para dar respuesta al objetivo uno se utilizó la estadística descriptiva a través de frecuencias y proporciones y estimaciones puntuales con intervalos de confianza al 95\%, así mismo se aplicó la prueba de Chi cuadrada de Pearson y calculo de riesgo a través de Razones de Momios (OR). Para la estadística inferencial se aplicó la prueba de Kolmogorov-Smirnov para conocer la normalidad de las variables continuas; en base a estos resultados se decidió la prueba no paramétrica para verificar las hipótesis de investigación, donde se utilizó el Coeficiente de Correlación de Spearman y la prueba para dos grupos independientes U de Mann-Whitney.

\section{RESULTADOS}

De acuerdo a datos sociodemográficos de los 558 participantes del estudio, se mostró que $50.5 \%$ fueron de sexo masculino, la mayoría soltero $(79.6 \%)$, vivían con sus padres $(52.7 \%)$ y $52 \%$ tenían un trabajo remunerado. En relación a la edad, los jóvenes tenían en promedio 22 años $(D E=5.03)$ y en promedio cuatro semestres cursados de carrera universitaria $(D E=2.85)$.

\section{Consumo de marihuana}

Se destaca que el 15.1\% (IC95\%, 12-18) ha consumido marihuana alguna vez en la vida, $5.4 \%$ (IC95\%, 3-7) en el último año y $2.2 \%$ (IC95\%, 1-3) en el último mes. El promedio de edad de inicio al consumo de marihuana fue a los 17.4 años $(D E=2.7)$ y en relación a los días de consumo en el último mes fue de 13 días en promedio $(D E=11.8)$.

En la Tabla 1 se observa la prevalencia de consumo de marihuana alguna vez en la vida por sexo, edad y estado laboral, don-

Tabla 1. Prevalencia de consumo de marihuana alguna vez en la vida por sexo, edad y estado laboral.

\begin{tabular}{|c|c|c|c|c|c|c|}
\hline \multirow[t]{2}{*}{ Variable } & \multicolumn{2}{|c|}{ Consumo de marihuana } & \multirow{2}{*}{$\begin{array}{c}\chi^{2} \\
\text { (valor } \mathrm{p})\end{array}$} & \multirow{2}{*}{ OR } & \multicolumn{2}{|c|}{ IC 95\% } \\
\hline & No $(\%)$ & $\mathrm{Si}(\%)$ & & & LI & LS \\
\hline \multicolumn{7}{|l|}{ Sexo } \\
\hline Femenino $\left(\mathrm{n}_{1}=276\right)$ & 94,9 & 5,1 & 42,5 & 6,17 & 3,38 & 11,27 \\
\hline Masculino $\left(\mathrm{n}_{2}=282\right)$ & 75,2 & 24,8 & $-0,001$ & & & \\
\hline \multicolumn{7}{|l|}{ Edad } \\
\hline$<20\left(\mathrm{n}_{1}=241\right)$ & 90,9 & 9,1 & 11,64 & 2,42 & 1,44 & 4,06 \\
\hline$\geq 20\left(\mathrm{n}_{2}=317\right)$ & 80,4 & 19,6 & $-0,001$ & & & \\
\hline \multicolumn{7}{|l|}{ Estado laboral } \\
\hline No trabaja $\left(\mathrm{n}_{1}=235\right)$ & 87,7 & 12,3 & 3,02 & 1,52 & 0,94 & 2,44 \\
\hline Trabaja $\left(\mathrm{n}_{2}=239\right)$ & 82,4 & 17,6 & $-0,082$ & & & \\
\hline
\end{tabular}


de se muestran diferencias significativas de las prevalencias de consumo de marihuana por sexo, se destaca mayor prevalencia en los hombres (24.8\%) que en las mujeres (5.1\%); los resultados del OR muestran que los hombres tienen hasta seis veces mayor posibilidad de consumo de marihuana que las mujeres. Por otra parte se muestra diferencias significativas por edad, se observa que los jóvenes universitarios mayores de 20 años de edad tienen mayor prevalencia, los resultados del OR muestran dos veces mayor posibilidad del consumo de marihuana en los mayores de 20 años de edad que en los menores de 20. En relación a las prevalencias por estado laboral no se encontraron diferencias significativas.

La Tabla 2 muestra la prevalencia del consumo de marihuana en el último año y en los últimos 30 días por área de estudio; antes

Tabla 2. Prevalencia de consumo de marihuana por área de estudio.

\begin{tabular}{lcccccc}
\hline \multicolumn{1}{c}{ Prevalencia } & \multicolumn{3}{c}{ En el último año } & \multicolumn{3}{c}{ En el último mes } \\
\cline { 2 - 7 } \multicolumn{1}{c}{ Área } & No $(\%)$ & Si (\%) & $\begin{array}{c}\chi^{2} \\
\text { (valor } \mathrm{p})\end{array}$ & No (\%) & Si (\%) & $\begin{array}{c}\chi^{2} \\
\text { (valor p) }\end{array}$ \\
\hline Sociales & 94,8 & 5,2 & 9,08 & 98,6 & 1,4 & 9,54 \\
Salud & 100 & 0 & $-0,028$ & 100 & 0 & $-0,023$ \\
Agropecuarias & 91,2 & 8,8 & & 94,1 & 5,9 & \\
Ingenierías & 90,3 & 9,7 & & 94,6 & 5,4 & \\
\hline
\end{tabular}

de aplicar la prueba de Chi cuadrada los 9 estratos correspondiente a las facultades se reagruparon en cuatro áreas de estudio considerando las áreas de conocimiento por el Consejo Nacional de Ciencia y Tecnología (CONACyT). Los resultados permiten observar que existe diferencias significativas $(p<.05)$ en la prevalencia del consumo de marihuana, se destaca mayor prevalencia de consumo de marihuana en los jóvenes de ingenierías y ciencias agropecuarias, y menor prevalencia en los jóvenes del área de sociales y de la salud.

\section{Intenciones del consumo de marihuana}

Los jóvenes que han consumido marihuana alguna vez en la vida $(\mathrm{n}=84), 65.5 \%$ (IC95\%; $55-76 \%)$, refirieron tener intención de volver a consumir, para aquellos que nunca han consumido marihuana $(\mathrm{n}=474) \quad 16.9 \%$ (IC95\%; 13\%-20\%) refieren tener intención en consumir marihuana. Con estos resulta- dos se muestra que existen mayores intenciones en los jóvenes que han experimentado el consumo de marihuana.

\section{Relación de la norma subjetiva para el con- sumo de marihuana con la conducta del consumo de marihuana}

Los resultados de la prueba de KolmogorovSmirnov mostraron que la variable norma subjetiva no presentó una distribución normal ( $D=5.97, p<0.05)$, en base a estos resultados se decidió el uso de la estadística inferencial no paramétrica para la verificación de las hipótesis.

Para dar cumplimiento al propósito del estudio y poner a prueba las hipótesis de investigación se presentan los siguientes resultados. El Coeficiente de Correlación de Spearman mostró relación positiva y significativa de la norma subjetiva con la intención del consumo de marihuana $\left(r_{s}=.224\right.$, $\mathrm{p}<0.001)$, es decir a mayor índice de norma 
subjetiva mayor es la intención para el consumo de marihuana.

En la Tabla 3 se muestra la prueba $U$ de Mann-Whitney para la norma subjetiva del consumo de marihuana sobre la intención de consumo de marihuana. Los resultados muestran diferencias significativas de las norma subjetiva por intención de consumo de marihuana $(U=20561.5, \mathrm{p}<.001)$, destacan- do medias y medianas más altas de la norma subjetiva en los jóvenes que refieren tener intención de consumir marihuana en un futuro comparado con los que no refieren intención. Con estos resultados se verifica la hipótesis de investigación uno que menciona: los jóvenes universitarios que muestran intención para el consumir marihuana muestran mayor percepción de norma subjetiva que los jóvenes

Tabla 3. Prueba U de Mann-Whitney para la norma subjetiva sobre la intención de consumo de marihuana.

\begin{tabular}{ccrcccc}
\hline Intención & $n$ & $\bar{X}$ & $M d n$ & $D E$ & $U$ & Valor dep \\
\hline SI & 135 & 15,85 & 9,72 & 18,93 & 20561,5 & 0,001 \\
NO & 423 & 6,99 & 4,72 & 10,77 & & \\
\hline
\end{tabular}

que no refieren intención.

Finalmente en la Tabla 4 se muestra la prueba U de Mann-Whitney para la norma subjetiva del consumo de marihuana sobre el consumo de marihuana. Los resultados muestran diferencias de la norma subjetiva por consumo de marihuana alguna vez en la vida $(U=28.71, \mathrm{p}<.001)$, en el último año $(U=13.96, \mathrm{p}<.001)$ y en los últimos 30 días $(U=5.78, \mathrm{p}<.001)$, destacando medias y medianas más altas de la norma subjetiva en los jóvenes que han consumido marihuana alguna vez en la vida, en el último año y en los últimos 30 días, comparado con los que

Tabla 4. Prueba U de Mann-Whitney para la norma subjetiva sobre el consumo de marihuana.

\begin{tabular}{lccccccc}
\hline Alguna vez en la vida & & $n$ & $\bar{X}$ & $M d n$ & $D E$ & $U$ & Valor de $p$ \\
& SI & 84 & 20,94 & 13,19 & 21,87 & 28713,5 & 0,001 \\
& NO & 474 & 7,05 & 4,16 & 10,43 & & \\
\hline \multirow{2}{*}{ En el último año } & & $n$ & $\bar{X}$ & $M d n$ & $D E$ & $U$ & Valor de $p$ \\
& SI & 30 & 30,53 & 33,33 & 24,59 & 13966,5 & 0,001 \\
& NO & 528 & 7,92 & 4,16 & 11,76 & & \\
\hline \multirow{2}{*}{ En los últimos 30 días } & & $n$ & $\bar{X}$ & Mdn & $D E$ & $U$ & Valor de $p$ \\
& SI & 12 & 35,76 & 37,5 & 18,88 & 5783 & 0,001 \\
& NO & 546 & 8,55 & 4,16 & 13,02 & & \\
\hline
\end{tabular}

no refieren consumo. Con esto se aprueba la hipótesis de investigación dos que menciona: los jóvenes universitarios que han consumi- do marihuana muestran mayor percepción de normas subjetivas que los jóvenes que no han consumido marihuana. 


\section{DISCUSIÓN Y COMENTARIO}

El presente estudio permitió aplicar empíricamente los conceptos de norma subjetiva, intención para el consumo y consumo de marihuana en un grupo de jóvenes universitarios de México.

Con respecto a la prevalencia de consumo de marihuana alguna vez en la vida fue de $15 \%$. Se mostró mayor prevalencia del consumo en el sexo masculino que en el sexo femenino, estimando 6 veces mayor la posibilidad de consumo de marihuana en los hombres que en las mujeres. Así mismo se observó una media de edad de inicio de consumo de marihuana a los 17 años. Estos resultados concuerdan con estudios previos realizados en México en población universitaria (16, 17). Sin embargo son superiores a lo reportado en población general de acuerdo a lo mencionado por la ENA 2008 (2), donde se reporta una media nacional de $4.2 \%$ de consumo de marihuana alguna vez en la vida. Esto puede deberse a que en esta región de México exista mayor oferta de la marihuana, dado que constantemente los medios de comunicación manifiestan la producción de ésta y pone de manifiesto que la población juvenil es una de las más vulnerables para el inicio de consumo de drogas.

Las diferencias de consumo por sexo indican que los hombres se muestran más vulnerables al consumo de marihuana. Esto se ha explicado por cuestiones culturales donde los hombres tienden a tener prácticas más riesgosas que las mujeres (16). Así mismo los resultados del presente estudio permiten afirmar que los hombres tienen mayor percepción de normas subjetivas, es decir perciben mayor tolerancia o permisividad de consumo de marihuana en su entorno y están más dispuestos a ceder a la presión de sus iguales. Otro dato relevante sobre el consumo fue que la media de edad de inicio concuerda con los autores anteriores $(16,17)$, lo que significa que los jóvenes universitarios tendieron al inicio de consumo de marihuana en la adolescencia.

De acuerdo a la edad los jóvenes mayores de 20 años tienen mayor prevalencia de consumo de marihuana, se muestra que es dos veces mayor la posibilidad de consumo de marihuana en los mayores de 20 años de edad que en los menores de 20 años. Estos datos concuerdan con la literatura revisada $(2,16,17)$, donde se explica que a medida que se incrementa la edad mayor son las oportunidades de consumo de cualquier droga, incluyendo la marihuana; esto posiblemente sea por tener mayor accesibilidad económicamente al consumo ya que más de la mitad de los jóvenes tienen trabajo remunerado, además es posible que en los jóvenes con mayor edad en su entorno social exista mayor disposición en ceder a la presión de sus amigos o compañeros. Por otro lado es posible que los jóvenes mayores tengan espacios donde no hay un seguimiento de la normativa legal ni ningún tipo de censura, por tanto se convierten en ambientes facilitadores para el consumo de marihuana.

En relación al área de estudio, se observó mayor prevalencia de consumo de marihuana en los jóvenes de ingenierías y ciencias agropecuarias, y menor prevalencia en los jóvenes del área de ciencias sociales y la salud. Esto es posible por las características de los jóvenes de ingeniería y agropecuarias, donde la mayoría eran de sexo masculino, y al tipo de formación académica y al conocimiento de los efectos nocivos, donde los jóvenes del área de la salud tienen más accesibilidad al conocimiento de los efectos nocivos del uso de drogas, lo que pudiera estar generando una baja tolerancia en su entorno hacia el consumo.

De acuerdo con las intenciones de consumir marihuana, los jóvenes que han consumido marihuana $(65.5 \%)$ tienen mayores intenciones que los que no han consumido (16.5\%). Este resultado concuerda con la literatura revisada $(10,13,14)$ y se apoya con los postulados de la Teoría de la Conducta 
Planificada $(7,8)$, que menciona que el factor que antecede la conducta es la disposición que tienen los sujetos para realizar la conducta en el futuro, y se puede explicar que el tener una experimentación previa al consumo de marihuana favorece las intenciones cuando la evaluación de los efectos de la experimentación son positivos.

Se mostró relación positiva y significativa de la norma subjetiva con las intenciones de consumo de marihuana, es decir a mayor disposición para ceder a la presión social para usarla mayor es la intención de consumo de marihuana, resultado que concuerda con estudios en el campo de las drogas donde se muestran coeficientes de correlación que van de 0.28 a 0.55 (10-14).

En el mismo sentido, los resultados permitieron probar la hipótesis uno que menciona: Los jóvenes universitarios que muestran intención para consumir marihuana muestran mayor percepción de norma subjetiva que los jóvenes que no refieren intención. Lo que permite verificar empíricamente uno de los postulados de la Teoría de la Acción Razonada (7) en jóvenes universitarios del norte de México, donde el conocimiento sobre las creencias específicas de lo que los amigos, compañeros y familiares piensan del consumo de marihuana influye en la intención de llevar a cabo dicha conducta, siempre en función de la motivación para complacerlos (7). Así mismo concuerda con un estudio previo realizado en adolescentes de educación media en México (14), lo que muestra que tanto adolescentes y jóvenes estudiantes son una población donde la referencia de los pares y compañeros influye en la disposición de consumir marihuana en el futuro.

En relación a la hipótesis dos planteada que menciona: Los jóvenes universitarios que han consumido marihuana muestran mayor percepción de norma subjetiva que los jóvenes que no han consumido. De la misma manera los resultados mostraron que los jóvenes que han consumido marihuana presentan norma subjetiva significativamente más alta que los jóvenes que nunca han consumido, lo que concuerda con lo reportado por la literatura internacional (10-12) y nacional $(13,14)$. El concepto de norma subjetiva permite explicar su influencia en conductas adictivas, y como se mostró con el constructo creencias normativas, el cual forma parte de las normas subjetivas, los universitarios perciben a los amigos y compañeros con mayor tolerancia hacia el consumo, es decir tienen un entorno favorable para el consumo de marihuana, lo que confirma la presión social o de amigos como un factor altamente relacionado con la conducta del consumo de marihuana. Ello pone de manifiesto que el concepto de norma subjetiva ha mostrado ser compatible con la cultura del joven mexicano, al probar uno de los postulados de la Teoría de la Conducta Planificada $(7,8)$ sobre la influencia de la norma subjetiva con el consumo de marihuana en jóvenes universitarios.

Finalmente, partiendo de la exigencia de la práctica actual de enfermería donde se plantea que los programas de intervención deben estar fundamentados en la práctica basada en evidencia científica, este trabajo ha tratado de probar conocimientos relevantes sobre el consumo de marihuana en jóvenes mexicanos, los resultados permiten enfatizar la pertinencia de abordar la norma subjetiva dentro de los diseños de intervenciones preventivas sobre el consumo de drogas.

\section{REFERENCIAS}

1. Guxens M, Nebot M, Ariza C, Ochoa D. Factores asociados al inicio del consumo de Cannabis: Una revisión sistemática de estudios de cohortes. Gac Sanit. 2007; 19(3): 1-9.

2. Consejo Nacional contra las Adicciones -CONADIC, Instituto Nacional de Salud Pública-INSP (MX). Encuesta nacional 
de adicciones. $1^{\text {a }}$ ed. Cuernavaca, Morelos, México: INSP; 2008.

3. Guzmán FR, Pedrao LJ. Personal and interpersonal risk factors in consumption of illicit drugs by marginal adolescents and young people from juvenile gangs. Rev Lat Am Enfermagem. 2008; 16(3): 368-74.

4. López KS, Costa ML. Antisocial Behavior Alcohol consumption by school adolescents. Rev Lat Am Enfermagem. 2008; 16(2): 299-305.

5. Latimer W, Floyd LJ, Karis T, Novotna G, Exnerova P, Brien M. Peer and sibling substance use: predictors of substance use among adolescents in México. Rev Panam Salud Pública. 2004; 15(4): 22532.

6. Benavides RA, Ceballo O, Colina BR, Esparza SE, García EG, Guzmán FR. et al. Autocuidado y estilo de vida saludable. Por la salud y el bienestar del universitario. $1^{\text {a }}$ ed. DF, México: Grupo Editorial Patria; 2009.

7. Ajzen I. The Theory of planned behavior. Organizational Behavior and Human Decision Process 1991; 50, 179-211.

8. Ajzen I, Fishbein M. Understanding attitudes predicting social behavior. Englewood-Cliffs, NJ: Prentice-Hall; 1980.

9. Elek E, Miller MD, Hecht ML. Influences of personal, injunctive, and descriptive norms on early adolescent substance use. Journal of Drug Issues. 2006; 31(1): 14772.

10. Norman P, Conner M. The theory of planned behaviour and binge drinking: Assessing the moderating role of past be- havior within the theory of planned behavior. Br J Health Psychol. 2006; 11(1): 55-70.

11. Saiz GJ. Estudio empírico de las variables de la teoría de la conducta planificada como factores de riesgo para el consumo de cocaína en tres grupos diferentes. Adicciones. 2009; 21(3): 187-94.

12. Umeh K, Patel R. Theory of planned behavior and ecstasy use: An analysis of moderator-interactions. Br J of Health Psychol. 2004; 9(1): 25-38.

13. Olivar AA, Carrero V. Análisis de la intención conductual de consumir Cannabis en adolescentes: desarrollo de un instrumento de medida basado en la teoría de la conducta planificada. Trastornos Aditivos. 2007; 9(3): 184-205.

14. Rodríguez KS, Díaz ND, Gracia GS, Guerrero HJ, Gómez ME. Capacidad predictiva de la teoría de la conducta planificada en la intención y uso de drogas ilícitas entre estudiantes mexicanos. Salud Mental. 2007; 30(1): 68-81.

15. Secretaría de Salud-(SS). Norma Oficial Mexicana NOM- 028-SSA2-1999 para la prevención, tratamiento y control de las adicciones. México, D.F. SS; 1999.

16. Chávez-Hernández AM, Macías-García LF, Páramo-Castillo D, Martínez C, Ojeda D. Consumo de drogas en estudiantes del nivel medio superior de la Universidad de Guanajuato. Acta Universitaria. 2005; 15(1): 13-21.

17. Font-Mayolas S, Gras ME, Planes M. Análisis del patrón de consumo de Cannabis en estudiantes universitarios. Adicciones. 2006; 18(4): 337-44. 